Schulnetzwerk SLECC (savoir lire, écrire, compter, calculer: Lesen, schreiben, zählen, rechnen können), das im Rahmen einer Öffnungsklausel die erarbeiteten Programme und Methoden für begrenzte Zeit umgesetzt hat; mittlerweile handelt es sich eher um ein Netzwerk einzelner Lehrkräfte oder Schulen, die im Rahmen der bestehenden Lehrpläne einzelne Aspekte von SLECC in den Unterricht integrieren. Aufgrund der intensiven Kontakte zu den mathematischen Forschern sind GRIP und SLECC recht einflussreich.

Danksagung.

Ich danke meinen Kollegen Thomas Bauer (Marburg) und Christian Kassel (Straßburg) für ihre kritische Durchsicht dieser Texte und viele wertvolle Bemerkungen.
Weiterführende Links

In französischer Sprache

Originalbericht: tinyurl.com/y8w3axe8

Homepage der Initiative SLECC (savoir lire, écrire, compter, calculer), die als eine mögliche Methode für Experimentiergrundschulen im Bericht erwähnt werden. Sie gilt als eher konservativ (nah an den Methoden, wie sie 1945-1970 verwendet wurden): www.instruire.fr, slecc.fr.

Blog von Luc Cédelle, Journalist bei der Zeitung Le Monde, der die Debatten um den mathematischen Anfangsunterricht gut wiedergibt: tinyurl.com/y9mhhao8

Webseite des Verbunds der IREM: www.univ-irem.fr

In englischer Sprache

Allgemeine Informationen zum französischen Schulsystem: tinyurl.com/y940ovmp

"Schools that build confidence" - Pressemitteilung vom September 2017 tinyurl.com/ydfjbqda

Wikipedia-Seite zum Mathematik-Unterricht in Singapur: en.wikipedia.org/wiki/Singapore_math

In deutscher Sprache

"Singapur - und jetzt werden alle kreativ“. Artikel aus der ZEIT o7/2017 tinyurl.com/y8t8xtcl

\title{
21 Maßnahmen für den Mathematik-Unterricht in Frankreich Die Hauptforderungen des Rapport Villani-Torossian
}

\begin{abstract}
Alle Maßnahmen und Empfehlungen werden im Originalbericht in einem oder mehreren separaten Absätzen erläutert und dann in sehr wenigen Worten zusammengefasst. Die Nummerierung und Kurzüberschriften der

Maßnahmen sowie die Überschriften der fünf „Blöcke“ entsprechen denen im Originalbericht. Die hier gegebene Beschreibung ist eine Synthese dieser Erläuterungen und Zusammenfassungen, weshalb die von den Autoren gewählten Kurzüberschriften nicht immer $100 \%$ passend erscheinen. Die wichtigsten Empfehlungen

wurden an der jeweils passenden Stelle bei den Maßnahmen mit aufgenommen. Entsprechend hat eine Auswahl stattgefunden, welche Empfehlungen und Aspekte in dieser Zusammenfassung vorkommen - und welche nicht. Wichtige französische Begriffe sind kursiv gesetzt.
\end{abstract}

1

Lehramtsausbildung

Eine Licence $(\mathrm{bac}+3)$ für das Grundschullehramt einführen, die alle dort unterrichteten Fächer und Fähigkeiten (lesen, schreiben, rechnen, zählen) abdeckt, dabei Spezialisierung auf ein Fach zulassen; als Fernziel sollten die Lehrer einer Grundschule auf diese Weise für jedes Fach mindestens einen darin besonders ausgewiesenen Kollegen haben. Alternativ eine "Mehr-Fach-Licence" (parcours pluridisciplinaire) anbieten.

In den klassischen Fächern der Licence Nebenfächer einführen; Mathematik als Nebenfach in allen geisteswissenschaftlichen Fächern zulassen.

Den Anteil der Fachdidaktik im „Lehramts-Master“ (Master MEEF) deutlich erhöhen.
2 Mathematik in den „Rep+"-Schulen ${ }^{1}$

Mathematik ab September 2018 als nationale Priorität in den „Rep+"-Schulen und ab 2020 in allen „Rep“-Schulen implementieren.

\section{Experimentierklauseln}

In der Sekundarstufe an ausgewählten Schulen (Ziel: ca. 200 Schulen) verschiedene fachdidaktische Methoden ausprobieren und wissenschaftlich solide evaluieren; Kandidaten hierfür sind Anpassungen der "Singapur-Methode“ und der SLECC-Vorschläge.

\section{$4 \quad$ Ausstattung mit Lernmaterialien}

Schulen mit mathematischen Modellen und Materialien nebst Anleitungen und Anwendungsbeispielen ausstat- 


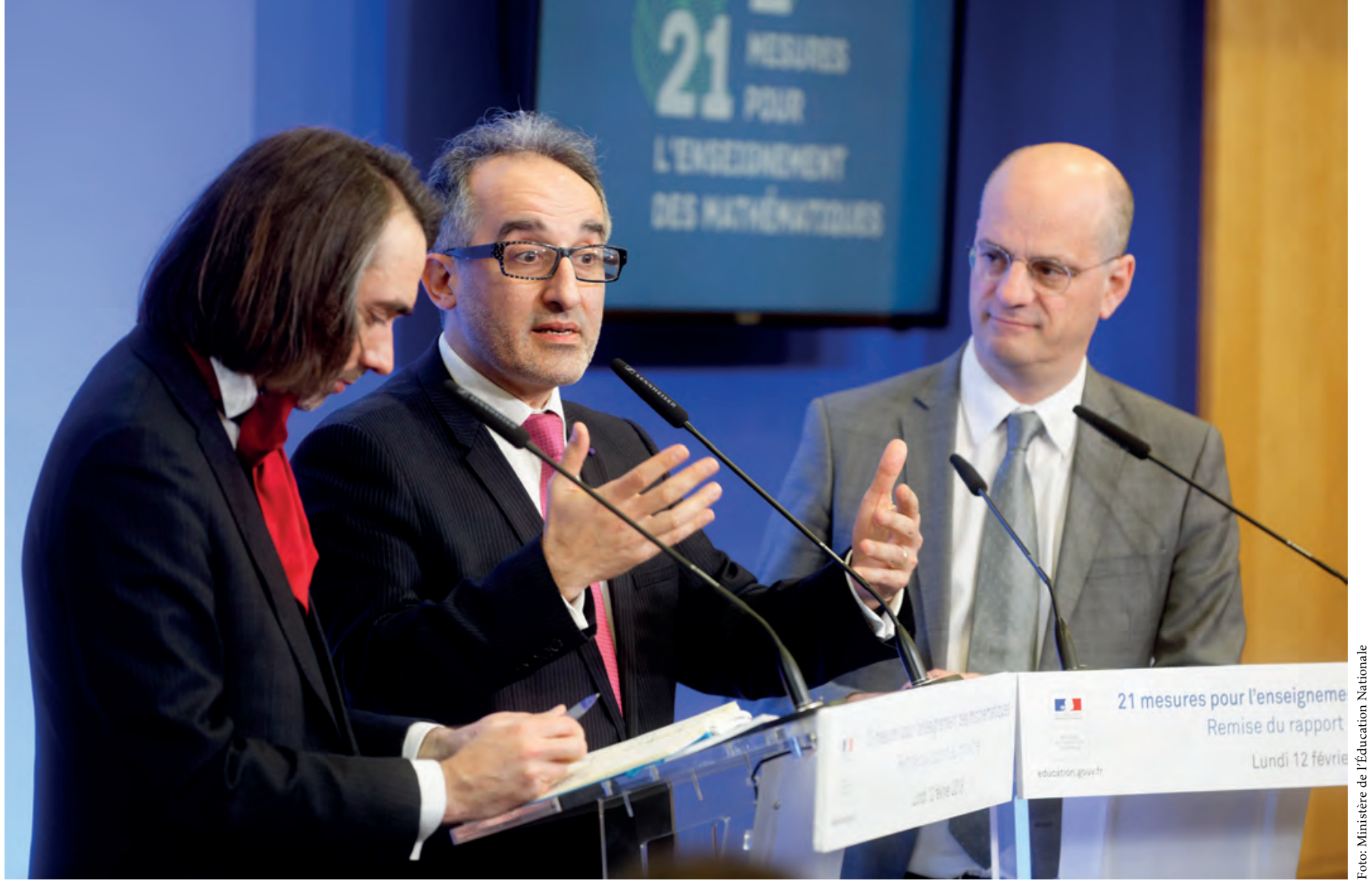

Cédric Villani, Charles Torossian und der Minister der Nationalen Bildung Jean-Michel Blanquer bei der Vorstellung des Berichts am 12. Februar 2018

ten, um das Begreifen reeller und virtueller Objekte zu fördern (etwa: geometrische Formen und Körper, Stecksysteme, Geobretter, Waagen, Messapparate, Spiele usw.).

\section{MATHEMATIK: EFFIZIENZ, SPASS UND EHRGEIZ FÜR ALLE SCHÜLER}

\section{Die Lernstufen}

$\mathrm{Ab}$ der Grundschule und bis zum Baccalauréat Mathematik erlernen, gegründet auf die drei Lernstufen

- spielerisches Begreifen und Experimentieren (manipulation),

- eigenständiges Formulieren (verbalisation),

- Abstrahieren (abstraction).

\section{Der Unterricht}

Den Mathematikunterricht ${ }^{2}$ neu ausrichten (,wieder ins Gleichgewicht bringen“ - rééquilibrer) unter besonderer Beachtung der drei Säulen:

- strukturierter Unterricht und angemessene Verschriftlichung, die den Schülern als Nachschlagewerk beim Lernen dienen kann und alle wesentlichen Konzepte und Ergebnisse logisch angeordnet enthält; dabei auf Verständlichkeit achten. Die Verschriftlichung neuer Inhalte sollte in der gleichen Unterrichtsstunde stattfinden.

- Beweise wieder sinnvoll in den Unterricht integrieren: Als Mittel zu mathematischem Verständnis, als Training für logisches und selbständiges Denken. Weiterhin soll auf einer Metaebene vermittelt werden, dass Beweise ein Weg zur objektiven Wahrheitsfindung sind und sie den Kern mathematischen Denkens und Schaffens bilden.

- explizites Üben und rituelles Anwenden gewisser Grundfertigkeiten.

Das „entdeckende Lernen“ sinnvoller einsetzen, um den Unterricht wieder effizienter zu gestalten sowie wieder aktiver auf das Wissen der Lehrkraft im Unterricht zurückgreifen.

Die affektive Dimension des Lernens explizit bei der Unterrichtsplanung berücksichtigen.

Eine positive Fehlerkultur entwickeln (Fehler zulassen und als wichtigen Teil des Lernprozesses ansehen).

Mehr Zeit in der Grundschule für Ausprobieren, Üben, das absolut sichere Erlernen des Zahlenraums zwischen 70 und $99 .{ }^{3}$

\section{Schulisches Umfeld (le périscolaire)}

Aktivitäten im schulischen Umfeld ausbauen, entsprechende Entlohnung und Anpassung der Stundenpläne. Spaß an und Beschäftigung mit Mathematik fördern durch AGs, Clubs, Wettbewerbe, Rallyes usw. Diese sollen nicht der Begabtenförderung dienen, sondern allen offen stehen. 
Bereits ab der Grundschule den Austausch mit anderen Schulfächern (v. a. im MINT-Bereich) ausbauen und das Herstellen von Querbezügen fördern; die Beziehungen zwischen Sprache und Mathematik (etwa: „einer, der, alle“ als Vorstufen der Quantoren) von Anfang an herausstellen.

9 „Wiederversöhnung“ der Schüler mit der Mathematik

Den Schülern Selbstvertrauen in Bezug auf Mathematik und ihren individuellen Lernfortschritt zurückgeben; die Mathematik aus der Ecke „Angstfach“ herausholen.

In der Sekundarstufe falls nötig einen gestuften Mathematikunterricht anbieten, etwa auf drei Leistungsniveaus: Förderniveau (hier „Wiederversöhnung“ genannt, im Sinne einer Wiederversöhnung mit dem Fach - réconciliation), mittleres Niveau (certification), Vertiefungsniveau (approfondissement).

10 Mathematische Projektarbeiten

In den Projektarbeiten der Sekundarstufe der Mathematik und Informatik einen wichtigen Platz einräumen; dabei v. a. mehr interdisziplinäre Projekte vergeben.

Den Informatikunterricht in der Schule ausbauen und mit dem Mathematikunterricht verknüpfen.

Die Lehrpläne auf innere Kohärenz überprüfen/ überarbeiten; Verstehen der Konzepte stärken; Ziele und Meilensteine klarer formulieren; geometrische Intuition fördern und in der Sekundarstufe mehr verwenden; Elemente der diskreten Mathematik in die Lehrpläne aufnehmen.

\section{ZAHLEN UND RECHENFERTIGKEITEN}

\section{Bedeutung der Zahlen und Grundrechenarten}

Heranführung an die vier Grundrechenarten ab der 1. Klasse. Größenordnungen und Maße sollen dabei besonders hervorgehoben werden.

\section{Automatismen fördern}

Rechenfertigkeiten durch häufiges Üben festigen (Kopfrechnen, Überschlagsrechnungen usw.), um später auch anspruchsvolle und motivierende Aufgaben bearbeiten $\mathrm{zu}$ können. Dabei auf Verständlichkeit achten und stupides Anwenden von „Kochrezepten“ vermeiden (vgl. Anhang für ein Beispiel zum Divisionsalgorithmus).

\section{Meilensteine}

Meilensteine in der Beherrschung des Zahlenraums und der Rechenfertigkeiten definieren und diese dreimal pro Jahr mit stark standardisierten elektronischen Tests erheben. Wiederholungskurse für diejenigen Schüler, die im ersten Test eines jeden Schuljahres ein gewisses Niveau nicht erreichen. Zeitnahe Rückmeldungen an die Eltern.

\section{WEITERBILDUNG UND VERNETZUNG}

\section{4 "Mathematischer Referent"}

Austausch unter den Lehrern fördern, insbesondere zwischen Berufsanfängern und erfahrenen Lehrern (équipes de mathématiques). In jedem Schulbezirk das Amt eines „Mathematischen Referenten“ einführen, der den Austausch koordiniert und fördert.

\section{Weiterbildung}

Die Weiterbildung für Lehrer aller Schulformen im Umfang deutlich ausweiten; gemeinsame Zeiten in den Unterrichtsplänen freihalten, Lehrer mit besonderen Kenntnissen (etwa aufgrund von Weiterbildungen oder der Hochschulausbildung) identifizieren und zur Weitergabe der Kenntnisse in der Schule animieren (in Informatik ist dies bereits implementiert).

Bessere Koordination zwischen Schulen und ESPE; den Anteil der IREM an der Weiterbildung erhöhen; das Weiterbildungsangebot mehr an die Bedürfnisse aktiver Lehrer anpassen, insbesondere Ergebnisse der empirischen Forschung vermitteln.

\section{6 „Mathematik-Labore“ gründen}

Binnen drei Jahren an mindestens fünf weiterführenden Schulen „Mathematik-Labore“4 gründen als Schnittstelle zu den Universitäten, als Ort der Begegnung, der Zusammenarbeit, des Austauschs und der Weiterbildung; Freistellung der beteiligten Lehrer im Umfang von mindestens einer Stunde pro Woche.

Teilnahme am Erasmus+ Programm für Lehrkräfte innerhalb Europas, um andere Unterrichtstraditionen und -methoden kennenzulernen.

Möglichkeit/Pflicht zur Hospitation in anderen Schulen für alle Lehrer (Ziel: dreimal pro Jahr).

\section{ORIENTIERUNG UND EVALUIERUNG}

\section{Nationale Priorität}

Mathematik zur Nationalen Priorität ausrufen, um alle Beteiligten am „Bildungszyklus“ (Lehrer, Schulleiter, Dozenten usw.) für ein gemeinsames Ziel zu mobilisieren.

\section{Mathematik-Beauftragter bei der DGESCO}

Eine Stelle eines Mathematik-Beauftragten im Ministerium bei der DGESCO $^{5}$ schaffen; dieser ist für die langfristige Umsetzung und Evaluierung der hier vorgeschlagenen Maßnahmen zuständig. 
In der Lehramtsausbildung Gleichstellungsfragen in Bezug auf Mathematik mehr berücksichtigen (etwa: typische Rollenbilder, Berufswahl usw.); durch AGs, Clubs, Mentorenprogramme u. ä. Mädchen und Minderheiten gezielt für MINT-Aktivitäten gewinnen.

\section{Schulbücher}

Eine Kommission einsetzen, die Kriterien für die Qualität von Schulbüchern entwickelt und auf deren Grundlage eine Rangliste der am häufigsten verwendeten Schulbücher erstellt (viele im Gebrauch befindliche Schulbücher sind veraltet, d.h. entsprechen nicht den aktuellen Lehrplänen, oder decken nicht den gesamten Lehrplan ab lassen etwa Beweise weg, wo diese im Lehrplan explizit vorgesehen sind).

\section{Internetportal}

Erstellen und Pflegen eines Internetportals mit digitalen Unterrichtsmaterialen und deren möglicher Verwendung im Unterricht (etwa: dynamische Geometriesoftware, Computer-Algebra-Programme, einfache Programmieraufgaben; Methoden der KI verwenden, um individualisierte Aufgaben anbieten zu können). ${ }^{6}$

\section{Anhang}

Stellenweise Division nach angelsächsischem Vorbild

\begin{tabular}{|c|c|c|c|c|c|c|c|}
\hline & 4 & 3 & 2 & 1 & & 1 & \\
\hline- & 3 & 4 & 0 & 0 & \multirow[t]{6}{*}{2} & 0 & \\
\hline & & 9 & 2 & 1 & & 5 & \\
\hline & \multirow[t]{3}{*}{-} & 8 & 5 & 0 & & & \\
\hline & & . & 7 & 1 & & & \\
\hline & & - & 6 & 8 & & & \\
\hline & & & & 3 & & & \\
\hline
\end{tabular}

Als Beispiel für eine verständlichere Schreibweise der schriftlichen Division wird diese Variante der angelsächsischen Vorgehensweise gegeben.

Es soll 4321 : 17 berechnet werden. Dazu wird erst die höchste Stelle (hier: Hunderterstelle) berechnet und als „partieller 1ooer Quotient“ aufgeschrieben (Divisor geht 100,200,...,900 mal in den Dividenden - hier: 200 mal) und vom Dividenden abgezogen, analog berechnet man mit dem Rest jeden darauf folgenden partiellen Quotienten (10er Quotient: Divisor geht 10,20,.., 90 mal in den Rest, hier: 50). Das Ergebnis erhält man durch Addition der partiellen Quotienten, also $200+50+4=254$. Der Rest 3 steht links. Möchte man die Nachkommastellen berechnen, so kommt als nächster partieller Quotient 0,1 usw. Die Vorteile der Methode sind:

- Vorgehensweise ist für den Schüler in jedem Schritt verständlich (man rechnet $17 \times 200$ und zieht das Ergebnis, 3400, vom Dividenden $a b$ ),

- die Größenordnung des Ergebnisses ist von Anfang an klar und transparent (kein Verrutschen der Stellen möglich),

- kein unmotiviertes „Herunterholen“ der nächsten Stellen des Dividenden (der vollständige Rest des Dividenden erscheint deutlich in der Rechnung),

- Methode funktioniert unabhängig davon, wie viele Stellen der Divisor hat.

\section{Anmerkungen}

1. Erläuterung: „Rep“ und „Rep+“ (réseau d'éducation prioritaire) bezeichnen - in zwei Abstufungen - Netzwerke von Schulen, bei denen aufgrund gewisser Parameter (etwa: hoher Anteil an Sozialhilfeempfängern, hoher Anteil von Schülern mit Migrationshintergrund, schlechtes Abschneiden bei Vergleichsarbeiten) ein „hohes sozioökonomisches Problempotential“ diagnostiziert wurde und die aufgrund dieser Klassifizierung mehr Mittel (u. a. geringeres Lehrdeputat der einzelnen Lehrkräfte bei gleichzeitig höherer Zuweisung von Unterrichtsstunden an die Schule, zusätzliche Sozialarbeiter, maximal 12 Schüler pro Klasse in den ersten beiden Jahren der Grundschule) zugeteilt bekommen. Die Bezeichnung wurde 2014 eingeführt, das Konzept gibt es in verschiedenen Variationen seit 1981. 2. Wir empfehlen Lesern mit guten Französischkenntnissen die Lektüre der S. 22-26 des Originals, welche gut lesbar sind. Darin werden die (Hinter-)Gründe für diese Empfehlungen weiter ausgeführt. Dabei ist zu beachten, dass "le cours" auf Französisch zwei Dinge bezeichnen kann: Sowohl den Unterricht als Ganzes als auch die Unterrichtsnotizen der Schüler.

3. Dies bezieht sich auf eine Besonderheit der französichen Sprache: Als Überbleibsel des bei den Kelten und Normannen gebräuchlichen Zwanzigersystems wird 7o als "sechzig-zehn“ , 8o als „vier-zwanzig“ und 90 als „vier-zwanzig-zehn“ gesprochen. In der Schweiz und Belgien haben sich dagegen die Bezeichnungen septante, octante, nonante durchgesetzt.

4. Diese Idee geht bereits auf Émile Borel im Jahr 1904 zurück. 5. DGESCO (Direction Générale de l'Enseignement Scolaire): Eine der fünf Abteilungen des Nationalen Bildungsministeriums, die für Schulbildung zuständig ist (die anderen kümmern sich etwa um Hochschulen, Forschung usw.)

6. $\mathrm{Zu}$ einem solchen Portal könnte die Seite eduscol.education.fr/ maths ausgebaut werden.

Prof. Dr. Ilka Agricola, Philipps-Universität Marburg, FB 12, Mathematik und Informatik,

Hans-Meerwein-Straße/Campus Lahnberge, 35032 Marburg agricola@mathematik.uni-marburg.de

Ilka Agricola ist seit 2008 Professorin an der Philipps-Universität Marburg und wissenschaftliche Leiterin der Mathematischen Modellsammlung. Seit 2014 ist sie Dekanin des Fachbereichs Mathematik und Informatik. Sie forscht auf dem Gebiet der Differentialgeometrie und Spin-Geometrie. Zusammen mit Thomas Friedrich veröffentlichte sie Lehrbücher zur Elementargeometrie und Vektoranalysis. 